\title{
Gender Typed Advertisements and Impression Formation: The Role of Chronic and Temporary Accessibility
}

\author{
Gita Venkataramani Johar \\ Marketing Division \\ Columbia Business School \\ Page Moreau \\ Department of Marketing \\ Southern Methodist University \\ Norbert Schwarz \\ Department of Psychology \\ University of Michigan
}

\begin{abstract}
In this research, we tested the effects of chronic and temporary sources of accessibility on impression formation. Although some research suggests that chronicity amplifies temporary effects because of greater susceptibility to external primes, other research suggests that chronicity masks temporary effects because of redundance. We demonstrate in a thought listing study that in the domain of gender stereotypes, trait stereotypes may be routinely applied by those with a medium or high tendency to stereotype women, making external primes redundant. Based on this redundancy, we proposed that gender stereotypical primes will have little influence on subsequent judgments of those with a medium or high tendency to gender stereotype. In contrast, gender stereotypical primes will result in the classic assimilation effect for those with a low tendency to gender stereotype. We tested these propositions in the domain of female role portrayals in advertising and examined the effect of advertisements that feature women as homemakers (vs. do not feature women) on trait judgments of a target woman whose behaviors are ambiguously described. Results from 2 experiments show that, as predicted by the redundancy hypothesis, judgments of medium and high tendency to stereotype participants are not affected by advertisements portraying homemakers. Also as expected, judgments of low tendency to stereotype participants are assimilated to the homemaker prime. These results hold across tendency to trait stereotype (Experiment 1) and tendency to role stereotype (Experiment 2) and for trait judgments as well as gift choice. Theoretical and practical implications of these findings are discussed.
\end{abstract}

Prior research has found that female depictions in advertising can affect both judgments and behavior. For example, Rudman and Borgida (1995) primed participants with advertisements depicting women as sexual objects and found that men exposed to those advertisements (vs. control advertisements) were more likely to later indulge in sexist behaviors,

Requests for reprints should be sent to Gita Johar, Department of Marketing, Columbia Business School, Uris Hall 514, 3022 Broadway, New York, NY 10027. such as stereotyped information acquisition and sexualized behavior during a job interview with a female applicant. Presumably, the advertisements primed perceivers to categorize women as sexual objects and hence enhanced the accessibility and potential applicability of this stereotype even in an inappropriate context. This research examines the impact of advertisements that portray women in stereotypical roles and demonstrates that the effect depends on the overlap between the advertisement's portrayal and one's own gender stereotypes. This research extends our understanding of stereotyp- 
ing and adds to a growing body of literature that documents social side effects of advertisement content. As in Rudman and Borgida's study, our interest is in implicit stereotyping effects in which incidental exposure to advertising unconsciously (i.e., without the perceiver's awareness or intention) has a systematic effect on judgments of women (Greenwald \& Banaji, 1995).

From a practical perspective, examining priming effects in the advertising domain is especially relevant given that the average American watches $3.4 \mathrm{hr}$ of TV per day, which translates to exposure to at least $30 \mathrm{~min}$ of ads. Further, content analyses have found that advertising tends to feature women in stereotypical roles (i.e., as housewives) rather than as professionals (Bretl \& Cantor, 1988; Nowak, 1990; Signorielli, McLeod, \& Healy, 1994). Our research therefore focuses on the priming effects of ads that depict women as homemakers (i.e., stereotypical primes). Below we review the relevant literature on the additive versus interactive nature of chronic and temporary sources of accessibility and contrast two types of interactive effects.

\section{THEORETICAL FRAMEWORK}

A large body of literature in social cognition has demonstrated that information is interpreted in terms of the applicable concept that is most accessible at the time of encoding (for reviews, see Higgins, 1996; Schwarz, 1995; Wyer \& Srull, 1989). This accessibility principle is at the heart of numerous context effects that have been investigated in social cognition research. In these studies, researchers introduced contextual cues (i.e., primes) that temporarily increased the accessibility of a concept and observed that new information is interpreted in a manner consistent with this concept, provided that the concept is applicable to the information at hand (e.g., Higgins, Rholes, \& Jones, 1977; Srull \& Wyer, 1979). This results in assimilation effects on subsequent judgments, that is, judgments that are closer to the implications of the contextual prime than would otherwise be the case.

The influence of increased temporary accessibility can only be observed, however, when certain conditions are met. Highly accessible concepts only influence the interpretation of information on which they can be brought to bear, that is, information that is ambiguous (i.e., at least two alternative constructs are equally applicable to it) and lends itself to an interpretation in terms of the concept (e.g., Higgins et al., 1977). Research has also demonstrated that the effects of increased temporary accessibility may be influenced by the chronic accessibility of the construct/category for each individual (Higgins, 1996; Higgins, King, \& Mavin, 1982). Whereas some studies have found independent and additive effects of the two sources of accessibility (Bargh, Bond, Lombardi, \& Tota, 1986; Rudman \& Borgida, 1995; also see Higgins, 1989), others have documented interactive effects (Higgins \& Brendl, 1995).
Consistent with the additive explanation, Bargh et al. (1986) found that participants' construct-related impressions of a target were stronger both when the construct was primed (vs. not primed) and when it was chronically accessible (vs. not accessible), resulting in additive effects of chronic and temporary accessibility. Higgins and Brendl (1995) also found such an additive effect. In addition, Higgins and Brendl documented an interaction effect such that chronic as well temporary accessibility of a construct (e.g., conceited) have to be high for judgments of extremely vague behaviors to be assimilated to the accessible construct. Vague behaviors are described as those for which no construct is strongly applicable. In contrast, ambiguous behaviors refer to those for which at least two alternative constructs are equally applicable (Higgins, 1996). Despite its chronic accessibility, the construct may not be viewed as applicable and hence would not be used extensively without a temporary prime when the target is vague. Thus, both additive and interactive effects have been documented in the literature.

These additive and interactive effects suggest that contextual primes are more influential under conditions of high (rather than low) chronicity. Note, however, that these effects have been obtained under conditions in which one particular trait is primed, and the trait is directly applicable to a behavioral description. In contrast, gender stereotypical advertisements provide complex information about women as a social category and usually include information that bears on multiple traits and role behaviors. When facing an impression formation task, the perceiver may draw on the category representation to interpret the target person's behavior. If so, one may only observe an influence of the primes when the primes are more extreme than the perceiver's chronically accessible category representation and hence change the temporary category representation, or when the category representation is of low chronic accessibility to begin with. If the perceiver's existing category representation is both highly accessible and consistent with the information conveyed by the primes, we expected the primes to show little effect because their content is redundant with chronically accessible information that is likely to be drawn on independent of the primes (cf. Schwarz \& Bless, 1992). We refer to this hypothesis as the redundancy hypothesis and explain how it is likely to function in the domain of gender typed advertisements and impression formation below.

\section{Stereotypes and Impression Formation}

Knowledge accessibility effects of this type underlie the impact of stereotypes on person perception (Stapel \& Koomen, 1998). Stereotypes can be conceptualized as a set of concepts pertaining to a social category. Gender stereotypes are defined as beliefs that certain attributes differentiate women and men (Ashmore \& Del Boca, 1981), and research suggests that they have four different components (Deaux \& Lewis, 1983, 1984)-trait descriptors, physical characteristics, role behaviors, and occupational status. Further, each of 
these components has a masculine and a feminine version with masculine (feminine) components significantly more associated with males (females). Research has focused on trait characteristics and has documented that men are perceived as possessing agentic traits such as self-assertion and mastery more than women. Conversely, women are perceived as possessing communal traits such as selflessness and concern for others more than men.

When a stereotype is highly accessible, these concepts are applied to new information about category members, resulting in stereotype consistent judgments, that is, an assimilation of the target to the category (see Fiske \& Taylor, 1991, for a review). One contextual influence on the accessibility of stereotypes is exposure to stereotype consistent exemplars. Seeing advertisements that portray women as homemakers, for example, both activates and confirms the traditional gender stereotype, making it more likely that this stereotype is subsequently applied in evaluating other members of the category. This contextual influence, however, should be observed primarily for individuals who are unlikely to apply traditional gender stereotypes spontaneously, that is, individuals who are low in their chronic tendency to stereotype women. For individuals who are high in this tendency, traditional gender role stereotypes are likely to be chronically accessible, thus attenuating the impact of additional contextual primes. Although additive effects of chronicity and priming may be observed, we expected the domain that we examined, that of gender stereotypes, to be characterized by high levels of chronic accessibility, which should obscure the additional effects of priming.

Contextual primes may also result in contrast effects such as when individuals become aware of a likely contextual influence and attempt to correct for it (e.g., Lombardi, Higgins, \& Bargh, 1987; Martin, 1986; Strack, Schwarz, Bless, Kübler, \& Wänke, 1993). However, such awareness is unlikely given the multitude of advertising that surrounds people in their daily lives. Contrast effects have also been observed when individuals are exposed to extreme exemplars, which are atypical for their category (e.g., Herr, 1989; Herr, Sherman, \& Fazio, 1983; Kunda \& Oleson, 1997). In this case, the extreme, atypical exemplar serves as a standard of comparison relative to which other members of the category appear all the more typical. Again, advertisements featuring homemakers are unlikely to be extreme primes for anybody regardless of their priors. Finally, contrast effects may be observed when specific person exemplars are used as primes (Stapel \& Koomen, 1998); however, several exemplars are primed by advertisements and these exemplars are unlikely to all serve as a standard of comparison and evoke contrast effects. Therefore, we expected that advertisements priming gender stereotypes are likely to result in assimilative rather than contrast effects.

As this discussion indicates, we proposed that the impact of a highly accessible exemplar on judgments of other members of the category depends on the extent to which the exem- plar matches an individual's representation of the category. First, temporarily accessible exemplars that confirm an individual's chronically accessible category representation are unlikely to have much impact given that the two knowledge structures are highly redundant. Even when these exemplars are included in the category representation, their inclusion does not change the implications of the knowledge structure (for a more detailed discussion, see Schwarz \& Bless, 1992). Hence, exposure to advertisements featuring women as homemakers may be unlikely to influence subsequent judgments of individuals who hold traditional gender stereotypes. Second, temporarily accessible exemplars that are neither redundant with an individual's chronic category representation nor discrepant enough to be excluded from this representation result in changes in the category representation. In this case, the inclusion of these highly accessible exemplars in the category representation changes the implications of the category representation and affects subsequent judgments on which this representation is brought to bear. We hypothesized that this is most likely to be the case when individuals with a low chronic tendency to stereotype women are exposed to homemaker advertisements. Although these individuals may not spontaneously think of women as homemakers, being a homemaker is part of the role repertoire of most working women as well. Accordingly, exposure to homemaker advertisements may result in an updating of the category representation with subsequent assimilation effects on judgments of other category members.

We tested these propositions in three studies. The first is a pilot study that confirms the assumption that a stereotypical prime (i.e., one emphasizing gender-stereotypic traits) is redundant with the gender representations of those with a high tendency to stereotype. Two experiments then tested the effects of chronic and temporary sources of accessibility on trait judgments when the prime is redundant (vs. not redundant) with the chronic category representation.

\section{PILOT STUDY}

This study was designed to examine the chronic trait associations for the category "woman" among those with different levels of tendency to stereotype. We used a thought listing task to measure chronic trait associations (Devine, 1989). The goal of the study was to confirm the redundancy of the traditional female trait associations for those with higher levels of tendency to stereotype.

\section{Procedure}

Two hundred and one male and female students who were recruited via campus advertisements and paid for their time participated in this study. They first completed an open-ended thought listing task in which they were asked to list all the thoughts that came to their mind when they 
thought of the category woman. They then filled out the Deaux and Lewis $(1983,1984)$ chronic Tendency to Gender Stereotype Measure described next.

\section{Tendency to Gender Stereotype Measure}

The Deaux and Lewis $(1983,1984)$ scale asked participants to rate the likelihood that a woman has various traits on a scale ranging from 0 (extremely unlikely) to 100 (extremely likely). The scale was marked in intervals of 5 , with 5 anchor points specified on the scale (extremely unlikely, moderately unlikely, neither likely nor unlikely, moderately likely, extremely likely). Following Deaux and Lewis $(1983,1984)$, participants first responded to eight practice items (e.g., "How likely or unlikely is it that an average person has red hair?"). Next, participants rated the likelihood that a woman has eight feminine (i.e., communal; e.g., kind, emotional) personality traits. These traits were drawn by Deaux and Lewis $(1983,1984)$ from the Personal Attributes Questionnaire (Spence, Helmreich, \& Stapp, 1974), which has been shown to have good psychometric properties.

\section{Results}

Tendency to Stereotype score. The Tendency to Stereotype score was computed by averaging the ratings on the eight communal traits. Because of the exploratory nature of this study, we divided participants into three levels of tendency to stereotype-low (score $\leq 63.13, n=65$ ), medium (score > 63.13 and $<73.75, n=64$ ), and high (score $>73.75, n=68$ ). Rather than do a simple median split, we wanted to obtain a finer understanding of the content of participants' chronic categories. Creating three groups enabled us to observe the content of both the extreme as well as the moderate respondents.

Beliefs about women. Each association with the category woman (i.e., each thought) was coded by two independent coders into the following categories: (a) stereotype component (Deaux \& Lewis, 1983, 1984): trait, physical attribute, role/occupation, or belief (Devine, 1989); and (b) type: feminine (communal), masculine (agentic), or other. Thus, each thought received two codes (e.g., trait and masculine). These categories were defined using the Deaux and Lewis $(1983,1984)$ scale. The coders agreed on $81 \%$ of stereotype component categorizations and 74\% of type categorizations. All disagreements were resolved by discussion. Trait judgments were the primary focus of the next experiment. We therefore focus in the following on trait thoughts to illustrate the redundancy of communal traits with chronic stereotypes of medium and high tendency to stereotype individuals.

Total number of thoughts did not differ across tendency to trait stereotype conditions of low, medium, and high tendency to stereotype $(M \mathrm{~s}=8.81,8.77$, and 8.70 , respectively; $F<1$ ). Confirming the validity of the scale, results revealed that low tendency to gender stereotype participants reported fewer number of communal traits compared to medium- and high-tendency participants combined $(M \mathrm{~s}=1.55,2.14$, and 2.10 , respectively), $F(1,194)=3.38, p=.07$. Also confirming the validity of the scale and consistent with Devine (1989), low tendency to stereotype participants reported more beliefs than medium- and high-tendency participants combined ( $M \mathrm{~s}=2.66$ vs. 2.03 and 1.97, respectively), $F(1$, $194)=3.36, p=.06$, and fewer traits than medium- and high-tendency participants $(M \mathrm{~s}=3.17,4.19,4.15$, respectively), $F(1,194)=3.57, p=.06$. There were no differences in agentic (i.e., masculine) traits among the three groups ( $M \mathrm{~s}$ $=.40, .41, .32$ ). Further, all groups reported significantly more communal traits than agentic traits.

We found evidence suggesting that stereotypic trait primes would be redundant with the content of the chronic structures of the medium- and high-tendency participants. The proportion of total thoughts that are stereotypical trait thoughts was $25 \%$ to $26 \%$ for these groups compared to $17 \%$ for the low-tendency group, $F(2,176)=4.16, p<.05$; low versus medium, $F(1,176)=5.42, p<.05$; and low versus high, $F(1,176)=6.85, p<.05$.

\section{Discussion}

Results of the thought listing task indicate that low versus medium and high tendency to gender stereotype individuals differ in the degree to which they spontaneously use gender stereotypic traits to describe women. Trait judgments of women are likely to be chronically stereotypical for both medium and high tendency to stereotype participants. This finding suggests that an external stereotypical prime would be less likely to affect the judgments of medium- and high-tendency participants. However, trait judgments of women are likely to be affected by external stereotypical primes for low tendency to stereotype participants, for whom stereotypical traits are not as highly accessible. This proposition was tested in the next two experiments.

\section{EXPERIMENT 1}

Participants were undergraduate male and female students who responded to campus advertisements and participated in two different studies for a $\$ 20$ monetary incentive. At Time 1, they responded to the same tendency to gender stereotype measure as in the pilot study disguised as a survey of student opinions.

Two weeks after completing the Tendency to Gender Stereotype Measure, participants performed three additional tasks for three ostensibly unrelated projects. First, participants viewed and rated advertisements (which featured homemakers or no women) for an alleged marketing research study. Then, as part of a study purportedly on reading comprehension, participants read an ambiguous description of a 
target woman and rated her on different traits. Finally, they completed demand checks, recalled the advertisements they had seen, and provided demographic information. To minimize the possibility that participants would connect the prime (i.e., the advertisements) with the rating task, the last two tasks were administered by a different experimenter. Eighty-one participants completed the Tendency to Gender Stereotype Measure and the priming study.

\section{Stimulus Materials}

Advertisement rating task stimuli. Two videotapesstereotypical and control-were prepared to manipulate the type of advertisement prime. Each tape contained 20 advertisements. The stereotypical tape contained 16 commercials that depicted women as homemakers and, to reduce suspicion, 4 advertisements that did not depict women at all. The control tape contained 20 advertisements that did not feature men or women. The advertisements on each tape were separated by $6 \mathrm{sec}$ of blank tape during which time participants rated the advertisements on two Advertisement Liking scales. As explained below, we also controlled for product category, advertisement liking, and advertisement familiarity across the tapes.

Selection of advertisements. Advertisements on daytime and prime-time television were taped over a period of 2 weeks. We viewed the advertisements and selected a subset that fit the following criteria. First, we selected advertisements that each depicted a woman in a homemaker role (i.e., performing homemaker tasks). Then, we identified control advertisements in similar product categories (e.g., personal care).

We created four pretest tapes, each containing approximately seven advertisements of each type, holding the product category constant (e.g., stereotypical and control advertisements for cars would be on the same tape). Six sec of blank tape was interspersed between the advertisements. Approximately 12 participants watched each tape and rated the advertisements on the featured woman's role (homemaker vs. working woman), advertisement liking, and advertisement familiarity. We first selected advertisements that fit our criteria of featuring women as homemakers. If this role rating was satisfied, we picked an advertisement in the same product category that did not feature women to serve as the control. Once we had identified 16 such advertisements, we computed the average liking and the average familiarity across the 16 advertisements for the stereotypical and control types. We ensured that these variables did not differ across the conditions. Each of the final tapes consisted of the 16 advertisements of one type (stereotypical or control) and four control advertisements that were kept constant across the tapes.

The final tapes were once again pretested to ensure equal liking across the conditions. Twenty-four respondents participated in the pretest and were randomly assigned to the tape conditions. The mean liking across all advertisements on each tape did not differ by condition $(p>.7)$ and was slightly above the neutral point of the 5-point scale ranging from 1 (extremely disliked) to 5 (extremely liked) in both conditions $(M$ s stereotypical $=3.38$ and control $=3.27)$.

Trait rating task stimuli. Participants were asked to rate a target woman on her personality traits based on a written description. The description of the target woman was adapted from that used by Banaji, Hardin, and Rothman (1993). The paragraph contained behaviors weakly related to communal traits such as dependence (e.g., "doesn't have a car, so we took mine") embedded among neutral behaviors. The behaviors were pretested with participants from the same pool as those participating in the final experiment to ensure that each of the behaviors was perceived to be weakly related to communal traits. Further, based on prior research showing that priming effects occur only when the prime is perceived to be applicable to the target (cf. Banaji et al., 1993; Higgins, 1989), the description was modified so that it was ambiguous with respect to the target's employment and marital status.

\section{Procedure At Time 1}

Respondents participated in small groups in a study advertised as a survey of student opinions. As the cover story, participants were told that the study concerned students' attitudes and that different students would be asked to rate different demographic groups on various scales. In reality, all participants completed the Tendency to Stereotype Measure (Deaux \& Lewis, 1983, 1984). Participants were then randomly assigned to the two tape conditions and were contacted 2 weeks later to participate in the market research study.

\section{Procedure At Time 2}

Advertisement rating task. Respondents participated in groups of 2 to 8 for the first study, which was introduced as an examination of student reactions to various commercials. Participants were told that this study was part of a large market research study on the attitudes and consumer behavior of various demographic groups in the United States. They were also informed that they would see a series of commercials and, immediately after viewing each advertisement, they should rate it on the two scales provided.

Participants first completed some personal information on their demographics, TV watching, and shopping behavior to make the cover story credible. Then, participants viewed the stereotypical or control videotape depending on their condition. After watching each advertisement, participants rated the advertisement on two 5-point scales ranging from 1 (very bad) to 5 (very good) and ranging from 1 (extremely disliked) to 5 (extremely liked), respectively. At the end of the videotape, the experimenter noted that 30 min remained of the participants' scheduled hour and asked them to help out a fellow doctoral student on his Ph.D thesis. The experi- 
menter then left the room and the second experimenter immediately entered.

Trait rating task. In this allegedly unrelated second experiment, the male experimenter told participants that they would need to complete two short questionnaires. The first questionnaire was described as a study about how people form impressions of others from written information. Participants were told that each of them would read about a different man or woman and that the description came from a friend describing a recent encounter with this person (cf. Banaji et al., 1993). This cover story was used to preclude hypothesis guessing about the stereotyping aspects of the experiment. This questionnaire was in a different font from that used for the advertisement rating task to reinforce the notion that the two studies were unrelated.

Based on pretest results, participants were given 1 min 40 sec to read the target description of Pamela. They then rated Pamela on personality traits drawn from Banaji et al. (1993) on 11-point scales ranging from 0 (not at all) to 10 (extremely). Four traits were communal (cooperative, polite, sympathetic, and thoughtful), and four traits were irrelevant (cultured, educated, neat, and talented).

This cover story and other aspects of the procedure were pretested with 37 participants to ensure that they did not connect the primes (i.e., the advertisements) with the trait rating task. Results showed that participants did not perceive that their responses on the personality trait task were affected by the advertisements that they saw earlier on the tape $(M=$ 1.62 ), rated on a 5-point scale ranging from 1 (not at all) to 5 (very much so).

Recall. The experimenter then handed out the second questionnaire. Participants completed demand check questions about the purpose of the different studies and were asked to recall the brands that they had seen advertised.

\section{Results}

Tendency to Stereotype Measure. We computed a Tendency to Stereotype score for each participant by averaging the score on the eight communal traits. These scores were used to divide participants into three groups of 25 to 30 participants each-low (scores $\leq 63.13, n=29$ ), medium (scores 63.13 to $73.13, n=27$ ), and high (scores $\geq 73.13, n=25$ ). Male and female participants did not differ in their tendency to stereotype $(p>3)$ and participant gender was not used as a factor in the analyses.

Target judgments. The four communal traits (cooperative, polite, sympathetic, thoughtful) had high reliability (Cronbach's $\alpha=.85$ ) and were therefore combined to form an index of stereotypical judgments. Mean communal trait ratings in the different conditions are shown in Table 1. The four irrelevant traits were analyzed separately because of low
TABLE 1

Trait Ratings of Pamela on Communal Traits

\begin{tabular}{|c|c|c|c|c|c|c|}
\hline \multirow[b]{3}{*}{ Advertisement } & \multicolumn{6}{|c|}{ Tendency to Stereotype } \\
\hline & \multicolumn{2}{|c|}{ Low } & \multicolumn{2}{|c|}{ Medium } & \multicolumn{2}{|c|}{ High } \\
\hline & $M$ & $S D$ & $M$ & $S D$ & $M$ & $S D$ \\
\hline Stereotypical & $6.20_{\mathrm{a}}$ & 1.73 & $7.10_{\mathrm{a}}$ & 1.62 & $6.65_{\mathrm{a}}$ & 1.87 \\
\hline Control & $4.78_{b}$ & 1.09 & $6.24_{\mathrm{a}}$ & 1.21 & $7.25_{\mathrm{a}}$ & .99 \\
\hline
\end{tabular}

Note. Means in the same column with different subscripts are significantly different at $p<.05$. Higher numbers reflect more stereotypic judgments.

reliability (Cronbach's $\alpha=.01$ ). These traits were not related to the prime and were not expected to reveal any effects of type of advertisement prime or tendency to stereotype. Results confirmed this expectation.

A $3 \times 2$ analysis of variance (ANOVA) with tendency to trait stereotype and type of advertisement as the independent variables revealed a significant main effect for tendency to stereotype, $F(2,75)=5.59, p<.01$, with the means showing the expected pattern of greater stereotyping of Pamela as the tendency to stereotype increased. Type of advertisement prime did not have a significant main effect $(p>.1)$. As expected, tendency to trait stereotype interacted significantly with type of advertisement prime, $F(2,75)=3.13, p<.05$. The pattern of means in the control condition confirms the validity of the low, medium, and high chronic accessibility categorization-mean ratings were lower in the low condition compared to the medium condition, $F(1,75)=14.87, p<$ .001 , and in the medium condition compared to the high condition, $F(1,75)=3.47, p<.07$.

As predicted, participants who were low in chronic tendency to stereotype women rated Pamela as more communal after they were exposed to the homemaker advertisements $(M$ $=6.20)$ compared to the control ads $(M=4.78), F(1,75)=$ $5.75, p<.05$. This effect reflects an assimilation of the target toward the primes. However, participants with a medium and high chronic tendency to stereotype were not influenced by exposure to the homemaker advertisements ( $p s>.1$; see Table 1 for means).

Demand checks. At the end of the Time 2 task, participants recalled the advertised brands. This served as an indirect measure of awareness of the prime (Lombardi et al., 1987). Overall awareness appeared low as desired ( $M=$ 6.9 out of 20) and was not significantly different across conditions. Participants reported that the TV commercials did not affect their ratings of Pamela $(M=1.4$ ) on a 5-point scale ranging from 1 (not at all) to 5 (very much).

Participants were also asked open-ended questions about the purpose of the questionnaire completed at Time 1, the advertisement rating study, and the trait rating study. None of them made connections between the different studies or guessed the true purpose of the experiment. 


\section{Discussion}

As expected, we found that the tendency to gender stereotype interacts with temporary contextual primes to affect judgments. Exposure to exemplars that matched medium and high tendency to stereotype participants' chronically accessible category representations (as verified in the pilot study) did not affect subsequent judgments of an ambiguously described target woman. This finding is consistent with the assumption that the information brought to mind by the advertisements was redundant with the information that control participants drew on spontaneously. In contrast, participants low in chronic tendency to stereotype women attributed more stereotypical traits to the target person after they saw advertisements that portrayed women as homemakers. This presumably reflects that these participants neither spontaneously think of women as homemakers (hence the difference to the control condition) nor consider homemakers extremely atypical for the category. Accordingly, they included the primed homemakers in their category representation, resulting in assimilation effects on subsequent judgments.

\section{EXPERIMENT 2}

Although the results of Experiment 1 are encouraging, some questions remain. First, the nature of the stimuli (TV advertisements) was such that the stereotypical and control advertisements may have differed along dimensions other than just the presence of women in stereotypical roles. For example, it could be argued that the stereotypical advertisements were not viewed by participants as portraying homemakers but as portraying women working around the house. To circumvent this criticism, we wished to create advertisements that clearly portrayed women performing behaviors previously identified as being more typical of women than men (Deaux \& Lewis, 1983). Second, the first experiment only examined the effect of participants' tendency to trait stereotype; yet as Deaux and Lewis (1983) pointed out, stereotypes also consist of roles, occupations, and physical characteristics. Would these other dimensions of tendency to stereotype have similar effects? Third, the first experiment did not examine whether the effect of the prime would carry over to variables other than trait judgments. This last issue is particularly important because of the absence of an effect for high chronic accessibility participants. It could be argued that the task used in the first study was calibrated so that either temporary or chronic accessibility would be sufficient to produce an effect. Experiment 2 was designed to address these issues.

The goal of this experiment was to replicate the findings of Experiment 1 using more controlled stimuli and the Tendency to Role Stereotype Scale (vs. the Trait Stereotype Scale used in the previous study). Further, additional dependent measures were included. Fifty-nine male and female stu- dents participated during a regularly scheduled class for partial course credit.

\section{Stimulus Materials}

Advertisement rating task stimuli. We created five print advertisements that displayed women in stereotypical roles (vacuuming, cooking, washing the dishes, flower arranging, and doing laundry) by photographing the same female model performing the different tasks. The roles were adapted from Deaux and Lewis (1983). We created eight control advertisements featuring the same background but without the model. Participants were randomly assigned to the stereotypical (five stereotypical advertisements plus three control advertisements) or control advertisements (eight control advertisements) conditions.

Trait rating task stimuli. Participants read about and rated the same target woman, now called Donna, on several traits as in Experiment 1. In addition, they rated several magazines on their appropriateness to be given as a gift to Donna. The magazines were selected using the Mediamark Research Inc. data on readership - six magazines were primarily targeted at women (e.g., Family Circle), nine at men (e.g., Muscle and Fitness), and five equally to both men and women (e.g., National Enquirer).

\section{Procedure}

The cover story was that a new company called "Castle Keepers" needed help selecting a photo to include in their advertisement and that to get higher reliability, each photo was to be rated twice. Castle Keepers was described in the advertisement as a service company that took care of all household chores for a standard monthly fee. This enabled us to show pictures of various parts of a house with or without a woman performing some chores. The first task that participants performed was to rate each advertisement on the same two scales as in Experiment 1. Each advertisement was shown twice to ensure that the priming manipulation increased the accessibility of the stereotype. Unlike the previous study, participants were not asked for any demographic information prior to exposure to the prime to avoid unintentionally priming other aspects of the self.

After this priming phase, participants ostensibly moved on to a different study on impression formation. In reality, they read the same description as in the previous study; the woman was named Donna in this study. They then completed the appropriateness-for-gift scale for several magazines ranging from 1 (not at all) to 5 (extremely) and then rated Donna on the same trait scales as in the previous study ranging from 1 (not at all) to 10 (extremely). The next study asked participants to complete a tendency to role stereotype questionnaire. Participants rated (from 0 to 100 ) how likely it was that a woman performed each of four stereotypical roles (cooks the meals, does 
the laundry, tends to the house, takes care of children). The last questionnaire contained several manipulation and demand check questions. First, participants were asked the purpose of each of the studies that they had completed in that session. They then rated each of the roles portrayed in the stereotypical advertisements on typicality of the role for the average woman ranging from 1 (not at all typical) to 5 (extremely typical). Finally, participants provided demographic information and were debriefed and dismissed.

\section{Results}

Data were analyzed in the context of a 2 (type of advertisement prime-stereotypical vs. control) $\times 3$ (tendency to role stereotype - low, medium, high) design. The tendency to role stereotype distribution was used to create the three groups ( $n$ $=20$ per group) on this variable: low, $<71.25$; medium, 71.25 to 83.75 ; high, $>83.75$. All means are in Table 2 .

Manipulation and demand checks. None of the participants guessed that the studies were related or the true purpose of the experiment. Participants rated the roles portrayed in the five stereotypical activities to be extremely typical of women ( $M=4.17$ on a 5-point scale). The Tendency to Role Stereotype Scale and the categories created were validated in that typicality ratings differed significantly between the groups $($ low $=3.76$; medium $=4.15 ;$ high $=4.49 ; p$ s low vs. medium and medium vs. high $<.01$ ).

Effects of prime. The $2 \times 3$ ANOVA on appropriateness of the stereotypical magazines as a gift for Donna revealed only a significant interaction effect, $F(1,53)=3.09, p<.05$. Follow-up contrasts revealed that participants with low tendency to role stereotype in the stereotypical advertisement condition were more likely to rate these magazines as appropriate than participants with a low tendency to role stereotype in the control advertisements condition $(M \mathrm{~s}=3.67 \mathrm{vs} .3 .05)$, $F(1,53)=4.06, p<.05$. There was no effect of the prime for the medium- or high-tendency participants. In other words, the assimilation effect found among low tendency to stereotype par- ticipants in the previous study was replicated with different stimuli and a different dependent measure. There were no effects on the appropriateness of male-oriented magazines.

Trait judgments of Donna were combined as in the previous study and subjected to the $2 \times 3$ ANOVA. The only significant effect was a marginally significant interaction, $F(2$, $53)=2.53, p<.10$. Planned contrasts revealed that judgments of Donna were more stereotypical after exposure to the stereotypical primes for low tendency to role stereotype participants $(M s=7.17$ vs. 6.19$), F(1,53)=3.49, p<.07$. None of the other contrasts were significant. Again, results from the previous study are replicated.

\section{DISCUSSION}

Results from the two experiments reveal that, paradoxically, stereotypical primes have the greatest stereotyping impact on judgments and behaviors of those with a low tendency to stereotype. It is encouraging that this effect was replicated for two scales of tendency to gender stereotypes (traits and roles) and for two dependent measures (trait judgments and choice of magazine).

\section{Contributions}

Theoretically, this article makes three contributions to the literature on priming effects. First, we showed that role portrayals in advertising affect category representations and hence influence judgments. We demonstrated that priming effects need not be limited to trait primes; other dimensions of stereotypes (e.g., roles) can also prime related trait concepts. The finding that advertising can act as a prime is consistent with the idea that information about one component of gender stereotypes (e.g., roles) can influence inferences about other components (e.g., traits) in a systematic way (Deaux \& Lewis, 1983, 1984). Our research therefore documents the generality of priming effects.

Second, we contributed to the priming literature by testing the redundancy hypothesis. Specifically, prior research has

TABLE 2

Mean Trait Ratings of Donna and Gift Choices

\begin{tabular}{|c|c|c|c|c|c|c|}
\hline & \multicolumn{6}{|c|}{ Tendency to Stereotype } \\
\hline & \multicolumn{2}{|c|}{ Low } & \multicolumn{2}{|c|}{ Medium } & \multicolumn{2}{|c|}{ High } \\
\hline & $M$ & $S D$ & $M$ & $S D$ & $M$ & $S D$ \\
\hline \multicolumn{7}{|l|}{ Stereotypical ads } \\
\hline Appropriateness: female-oriented magazines & 3.67 & .49 & 3.15 & .64 & 3.54 & .57 \\
\hline Appropriateness: male-oriented magazines & 2.28 & .44 & 1.97 & .55 & 2.18 & .77 \\
\hline Trait ratings of Donna & 7.17 & 1.24 & 6.55 & 1.03 & 7.33 & .78 \\
\hline \multicolumn{7}{|l|}{ Control ads } \\
\hline Appropriateness: female-oriented magazines & 3.05 & .74 & 3.52 & .43 & 3.75 & .77 \\
\hline Appropriateness: male-oriented magazines & 1.86 & .54 & 2.29 & .65 & 2.00 & .83 \\
\hline Trait ratings of Donna & 6.19 & 1.24 & 7.18 & 1.07 & 7.18 & 1.01 \\
\hline
\end{tabular}


suggested that people with chronically accessible (vs. inaccessible) categories are more likely to be susceptible to the effects of external primes (Bargh et al., 1986; Higgins \& Brendl, 1995). We showed that this is not the case when external primes are redundant with the chronically accessible category representation. This research therefore provides support for the redundancy hypothesis (cf. Schwarz \& Bless, 1992) by demonstrating that stereotypical primes have no effect on impression formation of ambiguous targets if gender stereotypical traits are chronically accessible.

Third, the priming literature has used relatively sterile priming tasks such as sentence unscrambling to prime specific constructs in memory (cf. Banaji et al., 1993). Although some research has used exemplar primes (Moskowitz \& Skurnik, 1999; Stapel \& Koomen, 1998; Stapel, Koomen, \& Van Der Pligt, 1997), the primes have been presented in the form of words. We demonstrated similar effects using real TV commercials as priming stimuli (see also Rudman \& Borgida, 1995). The advertisements resulted in automatic stereotyping judgments; participants were not aware of the effect of the advertisements on their ratings.

\section{Limitations and Future Research}

Several limitations of this article should be mentioned and can help direct future research. With respect to the research on context effects, our results document the redundancy effect under very specific conditions of overlap between the chronic category and the prime. Research is needed to identify and test factors that are likely to lead to redundancy versus greater receptivity to the prime among high chronics. Methodological limitations of this research that deserve mention include the fact that our stereotypical prime could be viewed as a simple category prime. Although it could be argued that a prime with a category exemplar (i.e., a woman) can make different constructs accessible to people with different category representations, future research should disentangle the simple category prime from the role prime (e.g., using advertisements depicting women performing nonstereotypical role behaviors). Experiment 1 used 16 advertisments and Experiment 2 used 10 experiments to prime gender stereotypes. Future research should examine the extent of priming required in more ecologically valid manners. Another interesting issue concerns the manner in which individual tendency scales should be used. For all scales such as Need for Cognition, Need for Closure, and so forth, the frequency distribution of scores is likely to be very different in different populations. Dichotomizing the frequency distribution using a study done within a population creates categories based on a participant's relative position in his or her population. A participant with the same score could be categorized very differently when surveyed in a different (e.g., in the case of tendency to gender stereotype, a more traditional) population. One could argue that the same score should reflect the same degree of tendency to stereotype re- gardless of the population surveyed and call for splitting the study participants using predetermined points (e.g., the midpoint) in the scale. However, the issue is more complicated than that. For example, social desirability bias could operate differently in different survey populations and thus invalidate the a priori classification scheme. This is clearly an important issue and should be addressed in future research on measurement of individual differences in general.

\section{Implications}

Practically, this research suggests that advertising can have important implications on society. Our findings suggest that advertising depicting women in sex-typed roles is likely to activate stereotypic constructs of women resulting in stereotypic judgments and behavior. In a similar vein, Schwarz, Wagner, Bannert, and Mathes (1987) found that women exposed to advertisements featuring homemakers reported less favorable attitudes toward political participation than women not exposed to such advertisements. This finding also illustrates the classic assimilation effect given that political participation is a behavior inconsistent with traditional gender roles. Advertisements portraying homemakers can have unexpected negative consequences by instigating stereotypic judgments; paradoxically, this occurs among recipients with a low tendency to stereotype. We acknowledge that these discouraging findings are limited to the short-term effects of advertisements, and it is likely that advertising can build, reinforce, or mitigate existing stereotypes in the long run. This issue awaits more systematic research. In combination with earlier findings (e.g., Rudman \& Borgida, 1995; Schwarz et al., 1987), our results highlight the potential societal impact of advertising and we hope that they stimulate further research in this area.

\section{ACKNOWLEDGMENTS}

Page Moreau is now at the Department of Marketing, University of Colorado.

We thank Mahzarin Banaji and Bob Wyer for sharing their stimulus materials, Kay Deaux for sharing the Stereotyping Assessment Scale, and Carolyn Simmons for her comments on the article. We also acknowledge financial support from the Columbia Business School Research Fund to Gita Venkataramani Johar and a fellowship from the Center for Advanced Study in the Behavioral Sciences to Norbert Schwarz.

\section{REFERENCES}

Ashmore, Richard D., \& Del Boca, Frances K. (1981). Conceptual approaches to stereotypes and stereotyping. In David L. Hamilton (Ed.), Cognitive processes in stereotyping and intergroup behavior (pp. 1-35). Hillsdale, NJ: Lawrence Erlbaum Associates, Inc. 
Banaji, Mahzarin, Hardin, Curtis, \& Rothman, Alexander J. (1993). Implicit stereotyping in person judgment. Journal of Personality and Social Psychology, 65, 272-281.

Bargh, John A., Bond, Ronald N., Lombardi, Wendy J., \& Tota, Mary E. (1986). The additive nature of chronic and temporary sources of construct accessibility. Journal of Personality and Social Psychology, 50, 869-879.

Bretl, Daniel J., \& Cantor, Joanne. (1988). The portrayal of men and women in U.S. Television commercials: A recent content analysis and trends over 15 years. Sex Roles, 18, 595-609.

Deaux, Kay, \& Lewis, L. Laurie. (1983). Assessment of gender stereotypes: Methodology and components. Psychological Documents, 13, 25 (Ms No. 2583).

Deaux, Kay, \& Lewis, L. Laurie (1984). The structure of gender stereotypes: Interrelationships among components and gender labels. Journal of Personality and Social Psychology, 46, 991-1004.

Devine, Patricia G. (1989). Stereotypes and prejudice: Their automatic and controlled components. Journal of Personality and Social Psychology, 56, 5-18.

Fiske, Susan T., \& Taylor, Shelley E. (1991). Social cognition. New York: McGraw-Hill.

Greenwald, Anthony G., \& Banaji, Mahzarin R. (1995). Implicit social cognition: Attitudes, self-esteem, and stereotypes. Psychological Review, 102, 4-27.

Herr, Paul M. (1989). Priming price: Prior knowledge and context effects. Journal of Consumer Research, 16, 67-75.

Herr, Paul M., Sherman, Steven J., \& Fazio, Russell H. (1983). On the consequences of priming: Assimilation and contrast effects. Journal of Experimental Social Psychology, 19, 323-340.

Higgins, E. Tory. (1989). Knowledge accessibility and activation: Subjectivity and suffering from unconscious sources. In Jim S. Uleman \& John A. Bargh (Eds.), Unintended thought (pp. 75-123). New York: Guilford.

Higgins, E. Tory. (1996). Knowledge activation: Accessibility, applicability, and salience. In E. Tory Higgins \& Arie W. Kruglanski (Eds.), Social psychology: Handbook of basic principles (pp. 133-168). New York: Guilford.

Higgins, E. Tory, \& Brendl, Miguel. (1995). Accessibility and applicability: Some "activation rules" influencing judgment. Journal of Experimental and Social Psychology, 31, 218-243.

Higgins, E. Tory, King, Gillian A., \& Mavin, Gregory H. (1982). Individual construct accessibility and subjective impressions and recall. Journal of Personality and Social Psychology, 43, 35-47.

Higgins, E. Tory, Rholes, William S., \& Jones, Carl R. (1977). Category accessibility and impression formation. Journal of Experimental Social Psychology, 13, 141-154.

Kunda, Ziva, \& Oleson, Kathryn C. (1997). When exceptions prove the rule: How extremity of deviance determines the impact of deviant examples on stereotypes. Journal of Personality and Social Psychology, $72,965-979$.

Lombardi, Wendy J., Higgins, E. Tory, \& Bargh, John A. (1987). The role of consciousness in priming effects on categorization: Assimilation versus contrast as a function of awareness of the priming task. Personality and Social Psychology Bulletin, 13, 411-429.
Martin, L. Leonard. (1986). Set/reset: Use and disuse of concepts in impression formation. Journal of Personality and Social Psychology, 51, 493-504.

Moskowitz, Gordon, \& Skurnik, Ian W. (1999). Contrast effects as determined by the type of prime: Trait versus exemplar primes initiate processing strategies that differ in how accessible constructs are used. Journal of Personality and Social Psychology, 76, 911-927.

Nowak, Kjell. (1990). Magazine advertising in Sweden and the United States: Stable patterns of change, variable levels of stability. European Journal of Communication, 5, 393-422.

Rudman, Laurie A., \& Borgida, Eugene. (1995). The afterglow of construct accessibility: The behavioral consequences of priming men to view women as sexual objects. Journal of Experimental Social Psychology, 31, 493-517.

Schwarz, Norbert. (1995). Social cognition: Information accessibility and use in social judgment. In Edward E. Smith \& Daniel N. Osherson (Eds.), Thinking. An invitation to cognitive science (pp. 345-376). Cambridge, MA: MIT Press.

Schwarz, Norbert, \& Bless, Herbert. (1992). Constructing reality and its altematives: An inclusion/exclusion model of assimilation and contrast effects in social judgment. In Leonard L. Martin \& Abraham Tesser (Eds.), The construction of social judgments (pp. 217-245). Hillsdale, NJ: Lawrence Erlbaum Associates, Inc.

Schwarz, Norbert, Wagner, Dirk, Bannert, Maria, \& Mathes, Lucia. (1987). Cognitive accessibility of sex role concepts and attitudes toward political participation: The impact of sexist advertisements. Sex Roles, 17, 593-601.

Signorielli, Nancy, McLeod, Douglas, \& Healy, Elaine. (1994). Gender stereotypes in MTV commercials: The beat goes on. Journal of Broadcasting \& Electronic Media, 38, 91-101.

Spence, Janet T., Helmreich, Robert, \& Stapp, Joy. (1974). The personal attributes questionnaire: A measure of sex-role stereotypes and masculinity-femininity. JSAS Catalog of Selected Documents in Psychology, 4, 43 (Ms. No. 617).

Srull, Thomas K, \& Wyer, Robert S. (1979). The role of category accessibility in the interpretation of information about other people: Some determinants and consequences. Journal of Personality and Social Psychology, $37,1660-1672$.

Stapel, Diederik A., \& Koomen, Willem. (1998). When stereotype activation results in (counter)stereotypical judgments: Priming stereotype-relevant traits and exemplars. Journal of Experimental Social Psychology, 34, 136-163.

Stapel, Diederik A., Koomen, Willem, \& Van Der Pligt, Joop. (1997). Categories of category accessibility: The impact of trait concept versus exemplar priming on person judgments. Journal of Experimental Social Psychology, 33, 47-76.

Strack, Fritz, Schwarz, Norbert, Bless, Herbert, Kübler, Almut, \& Wänke, Michaela. (1993). Awareness of the influence as a determinant of assimilation versus contrast. European Journal of Social Psychology, 23, 53-62.

Wyer, Robert S., \& Srull, Thomas K. (1989). Memory and cognition in its social context. Hillsdale, NJ: Lawrence Erlbaum Associates, Inc.

Accepted by Dawn Iacobucci. 